\title{
EDUCAÇÃO EM SAÚDE PARA COMUNIDADES REMANESCENTES DE QUILOMBOS.
}

\author{
Camila Andrea Souza de Jesus ${ }^{1}$ \\ Dennis Soares Leite ${ }^{2}$ \\ Ana Luíza Ferreira Martins ${ }^{3}$ \\ Keila de Nazaré Madureira Batista ${ }^{4}$ \\ Maria José Aviz do Rosário 5
}

Eixo Temático: Gênero, Raça e Etnia.

Resumo: As comunidades quilombolas enfrentam inúmeras dificuldades em razão das condições precárias de vida, pela falta de efetividade de políticas públicas de inserção social e resgate de sua história, identidade e cultura. Dentre as necessidades dos quilombolas, busca-se a efetividade do exercício do direito à saúde. O trabalho tem o intuito de realizar um levantamento bibliográfico de artigos referentes a educação em saúde para comunidades quilombolas publicados nos últimos cinco anos. Após a leitura criteriosa da amostra foram selecionados 4 artigos, os dados retirados dos artigos e inclusos na revisão foram: objetivos, metodologia, resultados e conclusão. Foram encontrados poucos estudos na literatura sobre a temática educação em saúde para comunidades quilombolas. Dessa forma, mais estudos precisam ser realizados, pois a educação em saúde é uma ferramenta importante para orientar a comunidade e, ao realizá-la é preciso considerar as representações culturais da população alvo bem como buscar a construção do conhecimento visando nortear a população, de modo geral, sobre todos os fatores que estão relacionados e podem ser os prováveis causadores das enfermidades, a fim de formar cidadãos críticos e conscientes e não apenas transmitir informação. Atenção especial deve ser dada para populações específicas como os quilombolas, tanto para as ações de prevenção, diagnóstico e controle de doenças, como para as de promoção à saúde.

\footnotetext{
${ }^{1}$ Discente de Pedagogia, camilapcs@yahoo.com.br, Universidade Federal do Pará (UFPA) /Belém, Bolsista do Programa Conexões de Saberes, PAPIM.

${ }^{2}$ Discente de Fisioterapia, dennissoares7@gmail.com, Universidade Federal do Pará (UFPA) /Belém, Bolsista do Programa Conexões de Saberes, PROEX.

${ }^{3}$ Discente de Fisioterapia, luizanana20@gmail.com, Universidade Federal do Pará (UFPA) /Belém.

${ }^{4}$ Doutora em Doenças Tropicais, keila.madureira@gmail.com, Professora da Universidade Federal do Pará (UFPA) /Belém.

${ }^{5}$ Doutora em Educação, mrosario@ufpa.br, Professora da Universidade Federal do Pará (UFPA) /Belém. Coordenadora do Programa Conexões de Saberes.
} 
Palavras-chave: Quilombola. Educação em saúde. Comunidades vulneráveis. Prevenção primária. Conhecimento.

\begin{abstract}
Quilombo communities face many difficulties because of poor living conditions, due to lack of effectiveness of public policies for social inclusion and rescue of their history, identity and culture. Among the needs of the quilombolas, seeks the effectiveness of the exercise of the right to health. This work aims to carry out a literature review of articles relating to health education for quilombo communities published in the last five years. After careful reading of the sample were selected 4 articles, data taken from articles and included in the review were: objectives, methodology, results and conclusion. Few studies were found in the literature about the subject health education for quilombo communities. Thus, further studies are needed, because health education is an important tool to guide the community, and to do this is necessary to consider the cultural representations of the target population as well as seeking the construction of knowledge aiming to guide the population, generally, on all the factors that are related and may likely be the cause of diseases, to form critical and aware citizens, not just transmit information. Special attention should be given to specific populations like the Quilombolas, both for the prevention, diagnosis, control of diseases and for health promotion.
\end{abstract}

Keywords: Quilombola. Health Education. Vulnerable Communities. Primary prevention. Knowledge

\title{
INTRODUÇÃO/JUSTIFICATIVA
}

Conhecidas, atualmente, como comunidades quilombolas, os grupos populacionais remanescentes dos quilombos encontram-se espalhadas por todo Brasil. A Coordenação Nacional de Articulação das Comunidades Negras Rurais Quilombolas (CONAQ) aponta para a existência de mais de 3 mil comunidades no país. As comunidades quilombolas foram reconhecidas oficialmente pelo Estado brasileiro em 1988, principalmente com a afirmação de seus direitos territoriais por meio do Artigo 68 do Ato das Disposições Constitucionais Transitórias da Constituição (ADCTA) (FREITAS, 2011). 
As comunidades quilombolas enfrentam inúmeras dificuldades em razão das condições precárias de vida, pela falta de efetividade de políticas públicas de inserção social e resgate de sua história, identidade e cultura. Dentre as necessidades dos quilombolas, busca-se a efetividade do exercício do direito à saúde. A doença falciforme e a hipertensão arterial têm sido registradas com frequência nos dados coletados junto às comunidades quilombolas (FREITAS, 2011).

Nos últimos anos, a transformação desejada para a atenção à saúde no país busca o fortalecimento da Atenção Primária, o desenvolvimento de ações de saúde com ênfase na promoção e ampliação da assistência ambulatorial, de forma descentralizada. A Estratégia de Saúde da Família (ESF), eixo central da política de Atenção Primária à Saúde (APS) no país tem o potencial de interferir favoravelmente sobre indicadores de saúde da população, inclusive reduzindo internações por condições sensíveis ao cuidado primário. Uma das contribuições da promoção de saúde e da atenção primária de saúde é a educação que visa ampliar o entendimento sobre saúde, concorrendo para o processo em que a comunidade aumente a sua habilidade de resolver seus próprios problemas com competência e intensifique sua própria participação (VIEIRA, 2013).

\section{OBJETIVO}

Realizar um levantamento bibliográfico de artigos referentes à educação em saúde para comunidades quilombolas publicados nos últimos cinco anos.

\section{METODOLOGIA}

Para o desenvolvimento desta revisão da literatura sobre educação em saúde para comunidades quilombolas, foram realizadas buscas de literatura científica nas seguintes bases de dados on-line/portais de pesquisa: Pubmed/Medline, Scientific Eletronic Library Online (SCIELO) Literatura Latino-Americana e do Caribe em Ciências da Saúde (LILACS), BIREME, banco de teses e dissertações da Coordenação de Aperfeiçoamento de Pessoal de Nível Superior (CAPES)/Conselho Nacional de Desenvolvimento Científico e Tecnológico (CNPq). Os descritores, pesquisados na página eletrônica dos Descritores em Ciências da Saúde (DeCs) e expressões utilizadas durante as buscas nas bases de dados foram: quilombolas, educação em saúde, saúde de quilombolas, prevenção primária, conhecimento, comunidades vulneráveis, atenção em 
saúde de quilombolas e saúde da família. A análise seguiu critérios de elegibilidade previamente determinados. Adotou-se como critério de inclusão; (1) a língua: português, (2) o ano de publicação (artigos publicados de 2010-2015), (3) artigos que tratassem da educação em saúde para comunidades quilombolas tanto para crianças, adolescentes, adultos e idosos. Foram excluídos artigos: (1) que não abordavam sobre a educação em saúde para quilombolas (2) aqueles que os pesquisadores não tiveram acesso à versão completa, (3) Artigos publicados antes de 2010. Após a leitura criteriosa da amostra foram selecionados 4 artigos.

\section{RESULTADOS}

DESCONHECIMENTO DA HIPERTENSÃO ARTERIAL E SEUS

DETERMINANTES EM QUILOMBOLAS DO SUDOESTE DA BAHIA, BRASIL. CIÊNCIA E SAÚDE COLETIVA. (BEZERRA, 2015).

PROMOÇÃO DE SAÚDE EM POPULAÇÃO QUILOMBOLA NORDESTINA: ANÁLISE DE INTERVENÇÃO EDUCATIVA EM ANEMIA FALCIFORME. ESCOLA ANNA NERY. (MENESES, 2015).

VIVENCIANDO O RACISMO E A VIOLÊNCIA: UM ESTUDO SOBRE AS VULNERABILIDADES DA MULHER NEGRA E A BUSCA DE PREVENÇÃO DO HIV/AIDS EM COMUNIDADES REMANESCENTES DE QUILOMBOS, EM ALAGOAS. SAÚDE E SOCIEDADE. (RISCADO, 2010).

\section{EDUCAÇÃO EM SAÚDE EM COMUNIDADES QUILOMBOLAS. REVISTA GAÚCHA DE ODONTOLOGIA. (RODRIGUES, 2011).}

Segundo (BEZERRA, 2014): Situações de vulnerabilidade social em populações quilombolas são observadas pelas baixas condições socioeconômicas e nas desigualdades reveladas pelas prevalências de doenças crônicas, como hipertensão 
arterial (HA). Inserida no contexto das doenças mais importantes relacionadas às razões étnicas, a HA gera um grande impacto para saúde pública, devido à alta prevalência, baixo controle e consequentes repercussões cardiovasculares. (MINISTÉRIO DA SAÚDE, 2011).

Estudo anterior reportou uma prevalência de HA de 45,4\% em população quilombola, sendo mais frequente em idades mais elevadas, baixos níveis socioeconômicos, inatividade física e excesso de peso (BEZERRA, 2014). Fatores modificáveis relacionados ao estilo de vida têm sido frequentemente associados à HA e, apesar de estudados, ainda prevalecem os baixos percentuais de conhecimento, tratamento e controle da mesma, tanto em população desenvolvida, quanto em desenvolvimento.

Além da importância para saúde pública em identificar a prevalência de HA e os fatores associados em grupos vulneráveis, torna-se também relevante analisar o seu conhecimento, pois, quanto mais cedo o indivíduo conhece a condição de ser hipertenso mais precocemente podem ser iniciadas as ações objetivando evitar o aparecimento de complicações provenientes desse agravo.

Entre os problemas de saúde dos negros brasileiros ressalta-se a ocorrência de doenças de herança genética como a anemia falciforme (AF), esta é caracterizada por uma alteração na molécula de hemoglobina $(\mathrm{Hb})$, onde ocorre a troca da base nitrogenada adenina pela base timina codificando o aminoácido valina ao invés de ácido glutâmico, resultando na síntese da hemoglobina $\mathrm{S}$ (HbS), uma variável estrutural, ao invés da hemoglobina normal denominada HbA (MANFREDINI, 2010)

Em uma comunidade quilombola, onde a população é composta por indivíduos afrodescendentes que, muitas vezes, estabelecem casamentos entre si, se mantém elevadas as chances de ocorrência de AF, tornando-se necessárias estratégias de prevenção, educação em saúde e aconselhamento genético.

O Ministério da saúde do Brasil reconhece que a promoção de saúde é fundamental para ser utilizada na atenção primária, a partir da elaboração do Manual de Educação em Saúde e Autocuidado na Doença Falciforme, que visa à capacitação de profissionais de saúde da equipe multidisciplinar no âmbito da assistência a pessoas portadoras de doenças falciformes. Desse modo, esses profissionais podem fomentar e facilitar as ações de promoção em todos os níveis de atenção à saúde. (MINISTÉRIO DA SAÚDE, 2008). 
Intervenções na área da saúde e da educação que abordem a violência contra a mulher e, em especial, a mulher negra se fazem necessárias, considerando que a desigualdade entre homens e mulheres se constitui em fator de grande vulnerabilidade para as mulheres. Este cenário de desigualdade, no qual a violência é exercida, contribui para o surgimento de diversas enfermidades como as DST/aids, doenças psicológicas, agravamento de outras situações patogênicas além de contribuir para altas taxas de mortalidade.

Segundo (MINAYO, 2006), no que se refere à violência, "Estima-se que cause mais mortes as mulheres de 15 a 44 anos do que o câncer, a malária, os acidentes de trânsito e a guerra". Pesquisa realizada pela Organização Mundial de Saúde (OMS) aponta que o HIV/aids e a violência contra a mulher não só interagem como se reforçam (KRUNG, 2002). Nesse sentido, (OLIVEIRA, 2003) considera de imprescindível importância a inclusão de práticas de promoção e educação em saúde da população negra nas rotinas assistenciais e facilitação do acesso em todos os níveis do sistema de saúde. A busca de alternativas para o enfrentamento dessas questões é dever ético de todos aqueles que estão envolvidos em saúde, educação, visando contribuir para o desenvolvimento de ações de promoção da igualdade de gênero, de condições sociais e direitos e a plena saúde das mulheres negras.

A educação e a informação sobre os cuidados com a saúde bucal têm sido ressaltadas por diversos pesquisadores. $\mathrm{O}$ desconhecimento sobre cuidados necessários de higiene bucal representa um fator a ser considerado, uma vez que a informação, embora disponível nas grandes mídias, não chega a todas as camadas da população da mesma forma e, dificilmente, é apreendida de modo a produzir conhecimento e autonomia em relação aos cuidados com a saúde. A importância de programas odontológicos educativos, que levantem e interpretem as necessidades das populações de menor acesso aos serviços de saúde odontológicos precisa ser valorizada (PAULETO, 2004).

A educação em saúde é compreendida como o processo de transformação que desenvolve a consciência crítica das pessoas a respeito de seus problemas de saúde e estimula a busca de soluções coletivas para resolvê-los (BIS, 2004).

\section{CONCLUSÃO}


Foram encontrados poucos estudos na literatura sobre a temática educação em saúde para comunidades quilombolas. Dessa forma, mais estudos precisam ser realizados, pois a educação em saúde é uma ferramenta importante para orientar a comunidade e, ao realizá-la é preciso considerar as representações culturais da população alvo, bem como buscar a construção do conhecimento visando nortear a população, de modo geral, sobre todos os fatores que estão relacionados e podem ser os prováveis causadores das enfermidades, a fim de formar cidadãos críticos e conscientes e não apenas transmitir informação. Atenção especial deve ser dada para populações específicas como os quilombolas, tanto para as ações de prevenção, diagnóstico e controle de doenças, como para as de promoção à saúde.

\section{REFERÊNCIAS}

BEZERRA, V. M.; CESAR, C. C. Desconhecimento da hipertensão arterial e seus determinantes em quilombolas do sudoeste da Bahia, Brasil. Ciência \& saúde coletiva, Rio de Janeiro, v. 20, nº 3, mar. 2015.

FALKENBERG, M. B.; MENDES, T. P. L.; SOUZA, E. M. Educação em saúde na saúde: conceitos e implicações para a saúde coletiva. Ciência \& Saúde Coletiva, Rio de Janeiro, v. 19, nº 3, mar. 2014.

FREITAS, D. A.; CABALlERO, A. D. Saúde e comunidades quilombolas: uma revisão da literatura. Revista CEFAC, Belo Horizonte, v. 13, p. 937-943, 2011.

GOMES, K. O.; REIS, E. A. Utilização de serviços de saúde por população quilombola do sudeste da Bahia, Brasil. Cadernos de Saúde Pública, Rio de Janeiro, v. 29, nº 9, set. 2013.

GOMES, K. O.; REIS, E. A. Utilização de serviços de saúde por população quilombola do sudeste da Bahia, Brasil. Cadernos de Saúde Pública, Rio de Janeiro, v. 29, nº 9, set. 2013.

LEITE, J. C. Saber formal e saber local: convergências e assimetrias. Ciência $e$ cognição, Cuiabá, v. 17, no 2, p. 135-154, set. 2012.

MARQUES, A. S.; FREITAS, D. A.; CALDEIRA, A. P. Atenção primária e saúde materno-infantil: a percepção de cuidadores em uma comunidade rural quilombola. Ciência \& Saúde Coletiva, Rio de Janeiro, v. 19, nº 2, fev. 2014.

MELO, M. F. T.; SILVA, H. P. Doenças crônicas e os determinantes sociais da saúde em comunidades quilombolas do Pará, Amazônia, Brasil. Revista da ABPN, Pará, v. 7, $\mathrm{n}^{\circ} 16$, p. 168-189, mar/jun. 2015. 
MENESES, R. C. T.; ZENI, P. F.; OliveIRA, C. C. C. Promoção de saúde em população quilombola nordestina: análise de intervenção educativa em anemia falciforme. Escola Anna Nery, Rio de Janeiro, v. 19, nº 1, jan /mar. 2015.

RISCADO, J. L. S.; OLIVEIRA, M. A. B.; BRITO, A. M. B. B. Vivenciando o racismo e a violência: um estudo sobre as vulnerabilidades da mulher negra e a busca de prevenção do HIV/AIDS em comunidades remanescentes de quilombos, em Alagoas. Saúde e Sociedade, São Paulo, v. 19, nº 2, dez. 2010.

RODRIGUES, S. A.; LUCAS, M. G.; CERQUEIRA, S. T. S. Educação em saúde em comunidades quilombolas. Revista Gaúcha de Odontologia, Porto Alegre, v. 59, nº 3, p. 445-451, jul /set. 2011. 